\title{
Quantification of tip-broadening in non-contact atomic force microscopy with carbon nanotube tips
}

Meinander, Kristoffer; Jensen, Thomas N.; Simonsen, Soren B.; Helveg, Stig; Lauritsen, Jeppe Vang

Published in:

Nanotechnology

Link to article, DOI:

$10.1088 / 0957-4484 / 23 / 40 / 405705$

Publication date:

2012

Document Version

Peer reviewed version

Link back to DTU Orbit

Citation (APA):

Meinander, K., Jensen, T. N., Simonsen, S. B., Helveg, S., \& Lauritsen, J. V. (2012). Quantification of tipbroadening in non-contact atomic force microscopy with carbon nanotube tips. Nanotechnology, 23(40), - . https://doi.org/10.1088/0957-4484/23/40/405705

\section{General rights}

Copyright and moral rights for the publications made accessible in the public portal are retained by the authors and/or other copyright owners and it is a condition of accessing publications that users recognise and abide by the legal requirements associated with these rights.

- Users may download and print one copy of any publication from the public portal for the purpose of private study or research.

- You may not further distribute the material or use it for any profit-making activity or commercial gain

- You may freely distribute the URL identifying the publication in the public portal 


\title{
Quantification of tip-broadening in non-contact atomic force microscopy with carbon nanotube tips
}

\author{
Kristoffer Meinander ${ }^{1}$, Thomas N. Jensen ${ }^{1}$, Søren B. Simonsen ${ }^{2,3}$, Stig Helveg ${ }^{2}$, and Jeppe V. Lauritsen ${ }^{1}$ \\ ${ }^{1}$ Interdisciplinary Nanoscience Center (iNANO), University of Aarhus, Denmark \\ ${ }^{2}$ Haldor Topsøe A/S, Nymøllevej55, DK-2800 Kgs. Lyngby, Denmark \\ ${ }^{3}$ CINF, Department of Physics, Technical University of Denmark, DK-2800 Kgs. Lyngby, Denmark
}

\begin{abstract}
Date: 04/03 2012)
Carbon nanotube terminated atomic force microscopy (AFM) probes have been used for the imaging of $5 \mathrm{~nm}$ wide surface supported Pt nanoclusters by non-contact (dynamic mode) AFM in ultra-high vacuum. The results are compared to AFM measurements done with conventional Sitips, as well as with transmission electron microscopy (TEM) images, which give accurate measures for cluster widths. Despite their ideal aspect ratio, tip-broadening is concluded to be a severe problem even when imaging with carbon nanotube tips, which overestimate the cluster width by several times the nominal width of the nanotube tip. This broadening is shown to be caused by a bending of the carbon nanotubes, and not by pure geometrical factors, which coincidentally results in a significant improvement for relative height measurements of tightly spaced high aspect ratio structures, as compared to what can be achieved with geometrically limited conventional probes. Superior durability, however, stands out as the defining feature of carbon nanotube terminated probes, allowing them to give results with a greatly enhanced reproducibility.
\end{abstract}

\section{Introduction}

Since the discovery of carbon nanotubes (CNT) in the early 1990's [1], efforts have been made toward finding ever more innovative applications for their remarkable physical properties. The unique attributes of these nano-scale structures include an incomparably high tensional strength, a broad range of electronic transport properties, and the novel geometric feature of truly one-dimensional objects [2,3]. It is with these geometric properties, giving CNTs a naturally high aspect ratio, that they have attracted the interest of the scanning probe microscopy (SPM) community. 
In all fields of SPM, where topography and other surface properties are deciphered through the interactions of a sharp tip with the sample surface, tip geometry plays a crucial role. Tips with low aspect ratios, where the lateral dimensions are comparable to their lengths, will most often interact with a surface with a larger area of the tip apex. A minimization of this area of interaction, will both simplify the understanding of complex tip-surface interactions, as well as increase resolution of the measurements as a smaller part of the surface interacts with the tip. Geometrical constraints of SPM tips also play a major role in deciding which surfaces that can accurately be mapped, as a tip which is too wide will interact with the surface not only at its apex, but also with its sides, leading to an image artifact called "tipbroadening”. CNTs offer a close to ideal structure for the apex of an SPM tip, solving both of these aforementioned problems, as their aspect ratios can be near infinite, with very much larger lengths than widths.

Successful methods of attaching CNTs to the apex of Si-tips on atomic force microscopy (AFM) cantilevers, developed in laboratory environments [4-5], have recently been adapted for the commercial production of CNT-terminated AFM-probes. With this progress, the use of CNT-terminated tips has become more commonplace in various fields of AFM. Several recent studies, especially concerning tapping-mode AFM on biological systems, have presented results where the use of CNT-tips played a crucial role [5-7]. This success has spread to other fields of AFM, among others non-contact AFM (ncAFM) in ultra-high vacuum (UHV) environments. Being one of the most sensitive AFM techniques, ncAFM in UHV is most often concerned with high-resolution imaging, even up to the limit of atomically resolving surface structures. Long-range tip-surface interactions often complicate measurements of this type, when using conventional Si-tips with apexes that quickly become too blunt - a problem that possibly could be solved by the high aspect ratios and inherent stability of CNTs. A few nc-AFM studies have been attempted using this solution, however, with little success, as the high lateral flexibility of CNTs seemingly hinders true atomic resolution [8-12]. Among the positive results, nonetheless, it has been noticed that irreversible changes of the tip structure, commonplace in nc-AFM measurements, caused by undesired inelastic interactions between the tip and the surface, seldom occur with CNT-tips [7]. This, for AFM untypical lack of wear in frequently used tips, is also of great benefit, as the reproducibility of measurements is enhanced and guarantees comparable tip-surface interactions throughout a larger set of images.

In this study, we examine the suitability of CNT-tips for nc-AFM characterization of the dimensions of tightly spaced surface supported nanometer-sized Pt clusters, emphasizing both benefits and drawbacks of the method. Special attention is given to tip-broadening, an artifact in all AFM measurements, usually caused by a convolution of the geometry of the tip onto the imaged surface. With CNT-tips, however, this 
phenomenon occurs through a highly unique mechanism. Measurements are compared both to nc-AFM measurements done with conventional Si-tips, as well as with images acquired by transmission electron microscopy (TEM), a method superior in resolving the actual lateral dimension of nano-scale objects. Finally some simple models are proposed, explaining the interactions between the CNT-tips and the measured surfaces, and how these will affect the outcome of the nc-AFM measurements.

Measuring the true dimensions of high aspect ratio structures has always been tricky with AFM in ambient conditions, as both tip geometry and environmental contamination, such as thin films of condensed water, are superimposed on surface topography. With the use of CNT-terminated tips in UHV conditions, both of these limitations are removed.

\section{Experimental}

Sample preparation, nc-AFM, and TEM were all performed in separate vacuum chambers. In order to confirm that contamination during the transportation stage did not crucially affect the results, TEM measurements were performed both before and at an intermediate stage during nc-AFM measurements. Samples were annealed in oxygen during preparation to ensure that further oxidation did not change their properties over the long time period, of up to several weeks, during which they were used in measurements. Both TEM and nc-AFM confirmed that no major changes to the samples took place over this time period.

\subsection{Sample preparation}

The samples used in this study consisted of Pt particles dispersed on flat amorphous thin films of either $\mathrm{Al}_{2} \mathrm{O}_{3}$ or $\mathrm{SiO}_{2}$. The $\mathrm{Al}_{2} \mathrm{O}_{3}$ thin films were grown by electron beam (e-beam) evaporator-assisted deposition, whereas $\mathrm{SiO}_{2}$ was formed by plasma oxidation, onto $40 \mathrm{~nm}$ thick $\mathrm{Si}_{3} \mathrm{~N}_{4}$ windows, allowing for electron transparency, which in turn were supported by specially prepared $0.35 \mathrm{~mm}$ thick Si wafers. Details on the preparation of these sample supports can be found in Ref. [13]. Platinum was thereafter deposited on the thin films through an additional e-beam evaporation of a Pt target $(99.99 \%$ nominal purity). Once deposited, the Pt, initially covering the surface as irregularly shaped, crystalline islands, was thermally aged up to temperatures of $650{ }^{\circ} \mathrm{C}$ in a mixture of $0.2 \% \mathrm{O}_{2}$ in $\mathrm{N}_{2}$ with a total pressure of 1 bar and a flow of $120 \mathrm{ml} / \mathrm{min}$ by using a tube furnace (Carbolite CFT). During thermal ageing, the samples were heated by $5{ }^{\circ} \mathrm{C} / \mathrm{min}$, kept at the ageing temperature for a total of 3 hours, and then finally cooled down to room temperature, with a rate of ca. $-2{ }^{\circ} \mathrm{C} / \mathrm{min}$ for the first $200{ }^{\circ} \mathrm{C}$. The error in furnace temperature was estimated to be $\pm 7^{\circ} \mathrm{C}[14]$. 


\subsection{Non-contact Atomic Force Microscopy}

Non-contact atomic force microscopy was performed at room temperature in a UHV chamber with a base pressure below $1 \times 10^{-10}$ mbar, using a variable temperature beam deflection nc-AFM (VT-AFM/STM, Omicron GmbH, Taunusstein, Germany), described with better detail in [15]. To improve imaging results, frequency demodulation and cantilever oscillation control were performed with easyPLL-plus electronics (Nanosurf GmbH, Liestal, Switzerland). Before measurements, the samples were fixed on standard Ta sample plates and introduced into the UHV chamber through a vacuum load lock. No further sample processing was done. The actual nc-AFM images were then recorded in the topography mode, by keeping the mean frequency shift $(\Delta f)$, due to tip-surface interactions, constant relative to a preset frequency shift value and recording the feedback signal of the tip-surface distance control as the tip traced the surface. A voltage was applied to the tip, relative to the sample holder, $U_{\text {bias, }}$ which was adjusted to minimize the electrostatic forces arising from the contact potential difference [16].

The carbon nanotube tips were commercial AFM tips (CNT-NCH type, Nanosensors, Neuchâtel, Switzerland), with cantilever spring constants of about $42 \mathrm{~N} / \mathrm{m}$ and resonance frequencies of approximately $330 \mathrm{kHz}$. The CNTs attached to the ends of the Si-probes were single-walled, with radii of $0.6 \mathrm{~nm}$. TEM images of each individual CNT were supplied, allowing for confirmation of their quality and alignment relative to the Si-probe. For comparability reasons commercial Si-tips of the same cantilever type (SSS-NCH type, from Nanosensors), with nominal radii of about $2 \mathrm{~nm}$, were also used. During measurements the cantilevers were oscillated with amplitudes of $7-9 \mathrm{~nm}$, with typical Q-factors measured to be around 40 000. As is common for AFM measurements, the surface of the sample was raster-scanned, with the tip moving fast across the sample in one direction (fast scan direction), and then after a backward scan moving one step in the perpendicular direction (slow scan direction) before acquiring the following line of the image. Typical image sizes were $400 \mathrm{x} 400$ points, with scan speeds of about 0.2 lines/s. Drift was minimized by allowing the system to stabilize over a period of several hours before any data was collected. Typically, $U_{\text {bias }}$ was found to be fairly constant, with a value of about -0.5 $\mathrm{V}$, over the entire surface area of the samples. Active regulation of $U_{\text {bias }}$ during AFM image acquisition did not have any effect on the measured cluster heights.

All nc-AFM images were analyzed with the Scanning Probe Image Processor (SPIP) software package (Image Metrology A/S, Hørsholm, Denmark), by manually measuring the width and height of each particle in the images. Heights are given as the vertical distance from the top of each cluster to the surface of the substrate in the tilt corrected images, whereas particle widths are given as the full width at half of the maximum height (FWHM), as averaged over several directions across the center of the clusters. This 
is the typical convention when measuring particle widths from AFM images, as AFM probes usually cannot penetrate below any regions of a surface projected structure, but rather continue interaction with the widest part of the particle by other parts of the tip, causing so called tip convolution or tip-broadening. The reported errors for the measured averages are standard deviations.

\subsection{Carbon nanotube length measurements}

Before actual nc-AFM measurements were performed, the characteristics of each CNT-terminated probe were carefully determined. This analysis included both AFM cantilever specifications as well as the length of the actual carbon nanotube at the end of the probe. Fig. 1 shows the results of a typical CNT length measurement, during which Z-spectroscopy is performed on a flat surface. When the oscillating probe approaches the surface, initial contact between the end of the CNT and the surface will be noticed as a rapid decrease in the oscillation amplitude. The cantilever will eventually regain its oscillation, as the nanotube bends horizontally, and only when the actual Si-tip of the AFM probe touches the surface (noticed as a deflection of the cantilever), does the oscillation again decrease to zero. The total difference in $\mathrm{Z}$, i.e. the height, between the position of the initial decrease in oscillation amplitude and the position of the initial deflection of the cantilever is then the same as the distance between the apex of the CNT and the end that is fixed to the Si-tip. Typical lengths of CNTs were found to be between $50 \mathrm{~nm}$ and $200 \mathrm{~nm}$, where the most stable measurements were achieved with probes that had the shortest CNT lengths, whereas the longest CNTs proved to be entirely unsuitable for imaging our surfaces. The performance of the CNT-tips was in no way altered by these measurements.

\subsection{Transmission Electron Microscopy}

TEM images of the $\mathrm{Pt} / \mathrm{SiO}_{2}$ samples were acquired with a CM300 FEG SuperTwin transmission electron microscope (Philips/FEI Company), while images of the $\mathrm{Pt} / \mathrm{Al}_{2} \mathrm{O}_{3}$ samples were acquired by using an image aberration corrected Titan 80-300 SuperTwin transmission electron microscope (FEI Company). Both microscopes were operated at $300 \mathrm{kV}$ with an ultimate information limit of ca. $0.14 \mathrm{~nm}$ and 0.10 $\mathrm{nm}$, respectively. The TEM images were recorded with bottom-mounted CCD cameras at magnifications corresponding to pixel sizes of $0.13 \mathrm{~nm}$ and $0.09 \mathrm{~nm}$ in the images of $\mathrm{Pt} / \mathrm{SiO}_{2}$ and $\mathrm{Pt}_{2} / \mathrm{Al}_{2} \mathrm{O}_{3}$, respectively. Pt particle widths were measured from the TEM images using a circular approximation of their projected area. The measurements were performed automatically as described in Ref. [14]. The estimated measuring error is a systematic error of $10 \%$ of the particle diameters due to calibration of the TEM and a random error of $0.5 \mathrm{~nm}$ for all particle diameters due to the automatic image analysis. For particle sizes below ca. $2 \mathrm{~nm}$ in diameter, the error due to the automatic measurements increases and becomes systematic. For this reason the presented TEM data only include particle diameters above $2 \mathrm{~nm}$. 


\section{Results and discussion}

Actual nc-AFM imaging was initially found to be rather complicated, due to minor differences in the behavior of CNT-terminated probes, as compared to measurements with conventional Si-tips. When using conventional tips, one of the quickest ways to achieve stable measurements, before scanning parameters are properly optimized, tends to be by imaging at a rather large scale. Hence, the user is forced to image larger areas when initializing measurements, after which scan sizes can slowly be decreased, all the while adjusting imaging parameters, until the desired length scale has been reached. When using CNT-tips, imaging larger areas is completely futile during the first measurements. Stable scan conditions at the beginning of measurements were only found when small enough scan sizes were chosen. Once stable scanning conditions had been reached, then the scan area could slowly be increased until the proper size had been reached. The reason for this seems to be that the CNTs tend to "wobble" while the probe is oscillated far from the surface, a behavior that only ceases through the additional constraint of tip-surface forces. If initial scan sizes are too large, there will not be enough lateral constraint on the nanotube for it to stabilize.

\subsection{Comparison between widths acquired by nc-AFM and TEM}

Figure 2 shows a typical nc-AFM image of an $\mathrm{Al}_{2} \mathrm{O}_{3}$ surface covered with Pt nanoclusters, acquired with a CNT-terminated probe. Nanocluster morphologies were thoroughly investigated in a separate paper [14], showing that they adopted shapes closely corresponding to hemispherical caps. The average height of the clusters was calculated to be $4.1 \pm 0.8 \mathrm{~nm}$, and coupled together with their rather dense coverage this fairly high corrugation of the surface presents a very challenging system to accurately measure with conventional AFM tips. The inset shows an image of the same surface, at the same length scale, acquired with TEM. The difference between these two images is quite remarkable, although a simple counting of particles confirms that the areal density is in fact approximately the same. The largest, cloud-like, particles present in the nc-AFM image were later confirmed with TEM to be contaminants (as they were only visible in TEM after, and not before, the initial AFM measurements), deposited on the surface of the sample during transportation, and were therefore excluded from the average particle calculations.

As can be seen from the images in Fig. 2, the apparent broadening of the particles in the nc-AFM measurements is severe. This is not intuitively expected, as the high aspect ratio of the carbon nanotubes should give particle widths that are broadened only by the minute physical width of the tubes themselves, i.e., the single-walled diameter of $1.2 \mathrm{~nm}$. Fig. 3 shows histograms of the measured widths from both ncAFM and TEM images of Pt nanoclusters, on both $\mathrm{Al}_{2} \mathrm{O}_{3}$ (Fig. 3 a) and $\mathrm{SiO}_{2}$ (Fig. 3 b) surfaces. The insets in both graphs show the heights for Pt clusters on each surface. From these it can be seen that the 
average particle heights differ between clusters on the two surfaces, giving $4.1 \pm 0.8 \mathrm{~nm}$ average heights for Pt clusters on $\mathrm{Al}_{2} \mathrm{O}_{3}$, and $5.6 \pm 1.8 \mathrm{~nm}$ average heights on $\mathrm{SiO}_{2}$, even though there is only a small difference in their average widths according to TEM. Although the width and height distributions for the $\mathrm{SiO}_{2}$ supported clusters are somewhat more complex than what is suggested by using parameters from a simple Gaussian fit, the similarity between the distributions justifies a direct coupling between these measured values. The bin sizes for each of the histograms were optimized according to the FreedmanDiaconis rule [17], which appeared to give the best representation of the statistical data.

Contrary to cone-shaped Si-tips, where broadening scales with the size of the measured particles, a spherically capped CNT should cause broadening that rapidly saturates to a value equal to the diameter of the tube, when measuring increasingly larger particles. Because of this, the relative heights of particles of these sizes should have only little bearing on the increase in their measured diameters. When comparing the increases in the average particle widths, however, we see that this is not the case. For the case of the lower Pt clusters supported on $\mathrm{Al}_{2} \mathrm{O}_{3}$, the average cluster width is $5.1 \pm 1.0 \mathrm{~nm}$ according to TEM. This value is more than doubled, to $11.0 \pm 1.3 \mathrm{~nm}$, when measured with nc-AFM. The higher Pt clusters supported on $\mathrm{SiO}_{2}$, on the other hand, have widths that almost triple, when comparing the $5.6 \pm 2.0 \mathrm{~nm}$ average widths in the TEM images to the $14.3 \pm 2.6 \mathrm{~nm}$ widths in the nc-AFM images. Not only have the average widths increased, but the distributions themselves have grown wider for particles on both surfaces, further adding to the evidence that more than mere geometric factors affect the apparent width of the particles. Simply superimposing the physical width of an AFM probe on the measured width of a set of particles will increase the average, without affecting the spread of the distribution.

\subsection{Comparison between tip-broadening with CNT-terminated tips and Si-tips}

In order to get a comparable value for tip-broadening with conventional tips, nc-AFM was also performed on the same samples using regular Si-tips. In Fig. 4 a) line profiles from both slow scan $(\sim 0.05 \mathrm{~nm} / \mathrm{s})$ and fast scan ( $40 \mathrm{~nm} / \mathrm{s})$ directions across a single cluster, imaged with both a CNT-terminated tip (black lines) and a conventional Si-tip (grey lines), can be seen. Fig. 4 b) shows the forward scan and backward scan acquired topography images for the respective tips. The clusters imaged with either tip are not the same, although their heights are similar enough to assume that they have comparable geometries, a fact which is clearly supported by the findings in Ref. [14]. Typical for images taken with Si-tips, there is a difference in the width of the particle in the fast and slow scan directions, even though TEM images confirm that the particles are approximately hemispherical. Although this can sometimes occur for asymmetric tip shapes, the main reason for this is the difference in imaging speeds, as the feedback loop 
controlling the tip-surface distance has less time to react to changes in topography. Quite the opposite is seen for the CNT-tip, where the width of the cluster is slightly larger in the slow scan direction.

When further comparing the line profiles, another difference in behavior of the two tips also becomes apparent. In both fast and slow scan directions the tip-broadening appears more severe for the CNTterminated tip. The precise shapes of the profiles also differ, in that the Si-tip images a rather small apex, while almost immediately decreasing in height at a rather shallow slope. The CNT-tip, on the other hand, images a broader cluster apex, after which the height drops more quickly toward the level of the substrate. A result of this is that the imaged width at the base of the cluster can actually appear to be smaller when imaging with CNT-tips, although the FWHM is almost always larger.

Fig. 5 compares nc-AFM images acquired with CNT-tips and Si-tips, where the main image in Fig. 5 a) shows the topography given by a CNT-tip and the smaller image in Fig. 5 b) shows that of a Si-tip. The length scale in both images is the same. Fig. 5 c) shows the height profiles across lines marked in Fig. 5 a) and b), with the solid line going across clusters imaged with the CNT-tip, and the dashed line across those imaged with the Si-tip. What can be seen from this figure is the difference in the depth to which the two different tips can penetrate in trenches between clusters on the surface. The average peak-to-valley distance $\left(\mathrm{z}_{\mathrm{p}-\mathrm{v}}\right)$, calculated for a larger sample of clusters in these images is considerably larger for the CNT-tip, $4.0 \pm 1.0 \mathrm{~nm}$, as compared to that of a Si-tip, $3.2 \pm 1.4 \mathrm{~nm}$. This is perhaps somewhat surprising, as the average tip-broadening is larger for CNT-tips than for Si-tips.

In part, the differences in penetration depths can be explained by the discrepancies in the line profiles over clusters with the different types of tips, with heights decreasing faster at the edges of clusters when imaged by a CNT-tip. The most crucial difference, however, is illustrated in the smaller inset in Fig. 5 a), showing a magnified (x3) part of the CNT-acquired image, where the shapes of three clusters lying close to each other have been distorted. When examining the image in the inset, it becomes clear that the CNT in fact can penetrate deeper into trenches between clusters, giving them the appearance of being separated by only a thin line, mainly because the broadening of clusters somehow appears to decrease in the interface between closely spaced clusters. If this was not the case, broadening of these three clusters should have caused them to appear as if melted together into one big cluster. In order to explain this phenomenon, one must include another signal acquired during nc-AFM measurements, namely the dissipation signal.

\subsection{Dissipation signal}


Damping, or energy dissipation, can be measured as the magnitude of the signal that is used to keep the amplitude of cantilever oscillation constant. If dissipation increases, this means that energy has been lost from the oscillation, usually because of some type of inelastic interaction between the tip and the surface, which must be compensated for by a higher driving voltage of the signal that is used for cantilever excitation. In essence, this signal can be used to decipher dissipative reactions between the tip and the surface.

Dissipation is common in nc-AFM, when increasingly smaller tip-surface distances are used in order to improve imaging contrast. Eventually one will enter a regime where inelastic interactions occur rather frequently between the tip and the surface. One major difference between CNT-terminated tips and Si-tips can be noticed in this regime. When imaging on surfaces overpopulated by clusters, using a conventional Si-tip, what most frequently happens through dissipative interactions are, so called, “tip crashes”. I.e., the tip apex will irreversibly change, in a manner that usually broadens the tip, thereby worsening the resolution of images acquired with it. In our experiments, the Si-tips had a tendency to crash, and become irreversible damaged, so often while imaging these surfaces that a full sized scan was rarely completed. In comparison, full sized images were routinely acquired with CNT-terminated tips, not because they did not "crash", but rather because crashes did not affect them in any noticeable way and measurements could continue. This inherent stability is not surprising, considering that the irreversible destruction of the apex of a carbon nanotube is not very likely when only involving forces typical for nc-AFM measurements.

For imaging with CNT-tips a strong dissipation signal was always present while the topography signal gave a strong contrast. A typical example of the coupling between topography and dissipation can be seen in Fig. 6, where Fig. 6 a) and b) are the topography images during forward and backward scans, respectively, while Fig. 6 c) and d) are their respective dissipation signals. A striking feature of the dissipation signal is that it is at its strongest around the perimeter of each cluster. Occasionally when imaging surfaces with conventional Si-tips, one can get a strong dissipative signal from the front edge of a structure, if the feedback loop of the measurement does not correct tip-surface distances fast enough. In this case one would never see dissipation on the far edge, as the tip would be too slow in approaching the surface after passing the highest point of the measurement. For the case of CNT-terminated tips, dissipation is seen almost uniformly around ever point of the perimeter, in both fast and slow scan directions. What this means is that every time the CNT is close to the edge of a cluster, something occurs that removes energy from the oscillation.

In order to understand this one must consider what happens when a flexible nanotube, fixed only in one end, is lowered with its free end close to an inert Pt cluster on a surface. If the tube is lowered directly on 
top of the cluster van der Waals (vdW) forces should only attract the tube closer, thereby pulling it downwards. If, however, it approaches at some point near the side the cluster, those same vdW forces should inevitably force the tube to bend, and, if the nanotube is lowered far enough down, it will eventually touch the cluster. This situation, where the CNT approaches the surface near the edge of a cluster, is schematically illustrated in Fig. 7. In a complete oscillation cycle the CNT will be lowered toward the cluster when it is still in free suspension (Fig. 7 a), once forces are large enough it will bend toward the cluster (Fig. 7 b), and as vdW forces are exponentially higher for shorter distances, it will be dragged along the edge of the cluster as the tip finalizes its oscillation with an upward movement (Fig. 7 c). This dragging motion will cause friction that inevitably dissipates energy from the oscillation. Once the CNT is retracted far enough it will bend back to its initial straight configuration (Fig. $7 \mathrm{~d}$ ). A similar chain of events is clearly supported by Fig. 8 a), where line profiles from both topography (solid line) and dissipation (dashed line) signals are compared. Starting from the center position of the cluster, i.e., where the topography signal is at its highest, on the right-hand side of the graph, and following the dissipation signal toward the edge of the cluster, one can see a quadratic increase up until the edge of the cluster, where the topography signal is at its lowest (marked by the dotted line). At this point there is a sudden decrease in dissipation, which rapidly falls down to a zero level. The increase in dissipation is to be expected, if the distance through which the tube is dragged along the edge of the cluster, and hence the total friction, increases. This will be the case, as the initial point of contact between the CNT and the cluster is dependent on how much the tube has to bend to actually touch it, this point being lower down for a greater bending. In essence the dissipation signal traces the topography of the cluster.

\subsection{Models of CNT bending}

In Fig. 8 b) two simple models are compared to the actual nc-AFM topography profile across a typical cluster (solid line). These models, derived using the actual cluster shape based on TEM measured widths (sparsely dotted grey line), include a simple geometrical model of a flexible tube (dashed line) and a model that takes into account the balance of vdW forces (closely dotted line), both those between the nanotube and the cluster, as well as those between the nanotube and the surface.

Before going into detail with the models, one should examine the forces involved in bending a CNT. The force needed to bend one end of a CNT by the distance $x$ has previously been determined as (1)

$$
F_{\text {Bend }}=\frac{3 \pi\left(D_{o}^{4}-D_{i}^{4}\right) E}{64 L^{3}} x
$$

where $D_{o}$ and $D_{i}$ are the outer and inner diameters of the tube, respectively, $E$ is Young's modulus, experimentally determined to be approximately $1.2 \mathrm{TPa}$ [18-19], and $L$ is the length of the tube [20]. By 
using the values, $D_{o}=1.2 \mathrm{~nm}, D_{i}=0.8 \mathrm{~nm}$, and $L=50 \mathrm{~nm}$, for the CNT used to measure the profile in Fig. $8 \mathrm{~b}$ ), one can calculate the effective spring constant $k=F_{\text {Bend }} / x \approx 2.4 \cdot 10^{-3} \mathrm{~N} / \mathrm{m}$ for a bending of the tube. Comparing this value to the spring constant of the AFM cantilever (42 N/m), it becomes evident that the forces needed to bend the tube will be available in any interaction between the tip and a surface.

The first simple model, illustrated in Fig. 8 b) as the dashed line, only regards the CNT as a flexible tube, which bends, due to a force exerted at its apex, in such a way that at every point along the length of the tube the bending is equally strong, i.e. the strain on the tube is distributed evenly over its entire length. In other words the bending is uniform along the entire length of the tube, with the dashed line derived as the highest point of the AFM-probe, at any given lateral distance from the center of the cluster, at which the tube, subject to any possible bending, will touch the cluster. If the AFM-probe is any higher at each specific lateral point around the cluster, the bending CNT will swing past the cluster without touching it. This model actually gives a fairly good agreement with the experimentally measured nc-AFM curve, with a near perfect fit in shape at the top of the cluster, and with a slope that only slightly differs along the edges of the cluster. The difference between the model and the measurements, in the slope furthest away from the cluster edges, shows that the actual tube does not bend quite as much as an ideal flexible tube at these distances. The reason for this difference becomes very much understandable, as forces between the tube and the surface features come into play.

If the apex of the CNT is approximated as a sphere with radius $R_{1}$, the cluster on the surface as another sphere with radius $R_{2}$, and the surface as an endless plane, then the vdW forces between the CNT and the surface can be described by (2)

$$
F_{\text {surf }}=\frac{H}{12 \pi l^{2}} 2 \pi R_{1}
$$

and forces between the CNT and the cluster are similarly given by (3)

$$
F_{\text {clus }}=\frac{H}{12 \pi l^{2}} \frac{2 \pi R_{1} R_{2}}{\left(R_{1}+R_{2}\right)}
$$

where $H$ is the Hamaker constant and $l$ is the distance between the objects in question [21]. The sum of the components of (2) and (3) perpendicular to the surface, i.e., the force which affects the oscillation frequency of the AFM cantilever, is then given by $F_{\perp}=F_{\text {surf }}+\sin \varphi F_{\text {clus }}$, where $\varphi$ is the angle between an imaginary line connecting the centers of the tip apex and the cluster. Using typical values for the Hamaker constant, of $10^{-20}-10^{-19} \mathrm{~J}$ [22], together with length scales of a few nanometers for $l$, as well as the 0.6 $\mathrm{nm}$ radius of the CNT and the $5.8 \mathrm{~nm}$ radius of the cluster, we are left with total forces in the area of 0.1 - 
$1.0 \mathrm{nN}$, which seems reasonable for an nc-AFM interaction. Plotting a constant $F \perp$ curve then gives us the closely dotted line in Fig. 8 b), which follows the contours of the experimentally measured curve fairly well, at the same time as this curve lies within the limit for a bending of the tube allowing for the nanotube to make contact with the cluster at the lowest point of its oscillation cycle. The force perpendicular to the surface can then be calculated as $F_{\|}=\cos \varphi F_{c l u s}$, resulting in a somewhat smaller force that nonetheless is sufficiently higher than the forces in the pN-range needed to bend a CNT a few nanometers.

The complete interaction of the CNT-probe with the cluster covered surface can then be understood as follows. As the tip approaches the surface, it experiences a vdW attraction from both the clusters and the surface itself. In the vicinity around a cluster it feels a force in directions both perpendicular and parallel to the surface. The perpendicular force acts as a constraint on the oscillation frequency of the cantilever, thereby affecting the nc-AFM measured topography, while the force parallel to the surface will ultimately pull the nanotube into contact with the cluster, which can be noticed in the energy dissipation. The slightly lower topography signal, than what would be expected according to the total perpendicular force curve, can be explained by stochastic processes. As the tip position is taken further and further away from the center of the cluster, it approaches ever more closely to the surface with each downward movement. At these distances even a small bending of the nanotube toward the cluster, taking the apex further away from the surface, will result in a large decrease in the total interaction strength, thereby forcing the tip closer to the surface. Such an effect would also explain why there is a clear, although decreasing, signal from the dissipation even at distances slightly beyond the point of the lowest topography signal.

\section{Conclusions}

CNT-terminated probes have been used for nc-AFM imaging of Pt nanoclusters supported on thin films of $\mathrm{Al}_{2} \mathrm{O}_{3}$ and $\mathrm{SiO}_{2}$. Results have been compared to TEM images of the same surface and to nc-AFM images acquired with conventional Si-tips, showing that tip-broadening can be of great concern during measurements with CNT-tips. This broadening is dependent on both the physical properties of the CNTprobe itself, as well as the precise geometrical shape of the particles being imaged. In the two systems that were studied here, tip-broadening due to CNT-surface interactions was found to be more severe than tipbroadening caused by the geometrical convolution of conventional Si-tips, giving widths of particles that were more than double those measured by TEM.

Careful analysis of all data related to the nc-AFM measurements gives hints toward the mechanism of tipbroadening with CNT-tips. By comparing the experimental results with two different models for CNT- 
surface interactions, the reason for tip-broadening can be explained as a bending of the CNT apex toward protruding surface structures, driven by a simple vdW interaction. When the CNT apex is approached toward the surface at a lateral distance sufficiently far from the center of the cluster, the nanotube will bend and eventually make contact with the cluster, giving the impression of a much wider particle, and thereby also causing dissipation of energy as the tip is pulled off the cluster in the next phase of the ncAFM cantilever oscillation cycle. The final topography signal is the result of a combination of both the interaction landscape around the cluster, dictating how close that tip apex can approach the surface, as well as pure geometrical constraints, guiding the bending and eventual contact between the tip and the cluster. An efficient way of decreasing the bending of a CNT probe, and thereby decreasing the broadening of surface features, would therefore be a shortening of the CNT, which drastically increases its bending stiffness.

Despite the rather huge broadening effect, CNT-tips were also seen to give more accurate results for particle heights than Si-tips, as a balancing of bending forces in between closely spaced clusters guided the tip apex deeper than what a geometrically constrained Si-tip could penetrate. Together with the added durability of CNT-terminated tips, allowing for longer scan sessions, as well as more reproducible results, CNT-tips proved to be very well suited for studies of closely spaced supported nanoparticles. In addition to giving valuable topographical information, they can also serve as probes to study the interactions between CNTs and various surface supported structures.

\section{Acknowledgements}

The iNANO group gratefully acknowledges financial support from the Lundbeck Foundation, Haldor Topsøe A/S and the European Research Council (ERC Grant no. 239834, “Oxidesynergy” (JVL)).

\section{References}

1. S. Iijima, Nature 354 (1991) 56.

2. H. W. Zhu, C. L. Xu, D. H. Wu, B. Q. Wei, R. Vatjai, and P. M. Ajayan, Science 296 (2002) 884.

3. M. S. Dresselhaus, G. Dresselhaus, and Ph. Avouris (ed), Carbon Nanotubes: Synthesis, Structure, Properties and Applications (Springer Topics in Applied Physics vol 80, Springer, Berlin, 2001).

4. J. Martinez, T. D. Yuzvinsky, A. M. Fennimore, A. Zettl, R. García, and C. Bustamante, Nanotechnol. 16 (2005) 2493-2496. 
5. $\quad$ N. R. Wilson and J. V. Macpherson, Nature Nanotechnol. 4 (2009) 483-491.

6. R. M. Stevens, Mater. Today 12 (2009) 42-45.

7. C. V. Nguyen, K.-J. Chao, R. M. D. Stevens, L. Delzeit, A. Cassell, J. Han, and M. Meyyappan, Nanotechnol. 12 (2001) 363-367.

8. $\quad$ E. S. Snow, P. M. Campbell, and J. P. Novak, Appl. Phys. Lett. 80 (2002) 2002-2004.

9. $\quad$ E. S. Snow, P. M. Campbell, and J. P. Novak, J. Vac. Sci. Technol. B 20 (2002) 822-827.

10. D. Dietzel, M. Faucher, A. Iaia, J. P. Aimé, S. Marsaudon, A. M. Bonnot, V. Bouchiat, and G. Couturier, Nanotechnol. 16 (2005) S73-S78.

11. V. Barwich, M. Bammerlin, A. Baratoff, R. Bennewitz, M. Guggisberg, C. Loppacher, O. Pfeiffer, E. Meyer, H.-J. Güntherodt, J.-P, Salvetat, J.-M. Bonard, and L. Forró, Appl. Surf. Sci. 157 (2000) 269-273.

12. M. C. Strus, A. Raman, and C. V. Nguyen, Nanotechnol. 16 (2005) 2482-2492.

13. S. B. Simonsen, I. Chorkendorff, S. Dahl, M. Skoglundh, J. Sehestad, and S. Helveg, J. Am. Chem. Soc. 132 (2010) 7968-7975.

14. S. B. Simonsen, I. Chorkendorff, S. Dahl, M. Skoglundh, K. Meinander, T. N. Jensen, J. V. Lauritsen, and S. Helveg, J. Phys. Chem. C 116 (2012) 5646-5653.

15. G. H. Enevoldsen, A. S. Foster, M. C. Christensen, J. V. Lauritsen, and F. Besenbacher, Phys. Rev. B 76 (2007) 205415.

16. S. Gritschneder, Y. Namai, Y. Iwasawa, and M. Reichling, Nanotechnol. 16 (2005) S41-S48.

17. A. J. Izenman, J. Am. Stat. Soc. 86 (1991) 205-224.

18. $\quad$ M. M. Treacy, T. W. Eddesen, and J. M. Gibson, Nature 381 (1996) 678.

19. $\quad$ E. W. Wong, P. E. Sheehan, and C. M. Lieber, Science 277 (1997) 1971.

20. J. M.Kinaret, T. Nord, and S. Viefers, Appl. Phys. Lett. 82 (2003) 1287.

21. J. Israelachvili, Intermolecular and Surface Forces (Academic, San Diego, 1992); J. Mahanty and B. W. Ninham, Dispersion Forces (Academic, New York, 1976).

22. $\quad$ C. Argento and R. H. French, J. Appl. Phys. 80 (1996) 6081-6090. 


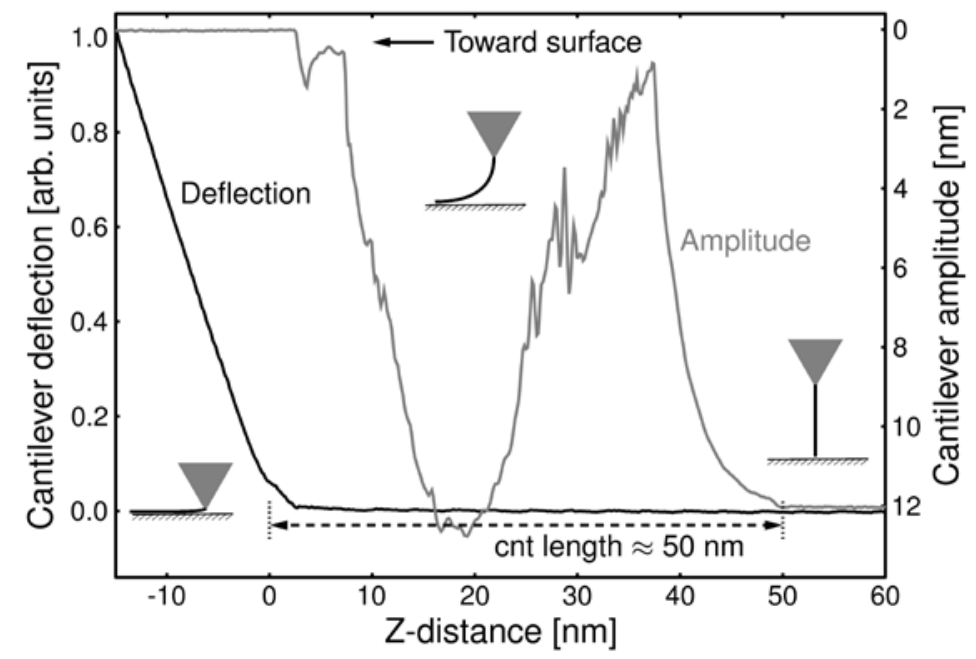

Figure 1: Carbon nanotube length measurement. Z-spectroscopy gives an initial decrease in the amplitude as the CNT contacts the surface (apparent from the inverse amplitude scale on the right-hand side of the graph), after which the cantilever regains its oscillation as the CNT bends on the surface. Only when the actual Si-tip touches the surface, causing a deflection of the cantilever, does the amplitude of oscillation decrease to zero again. 


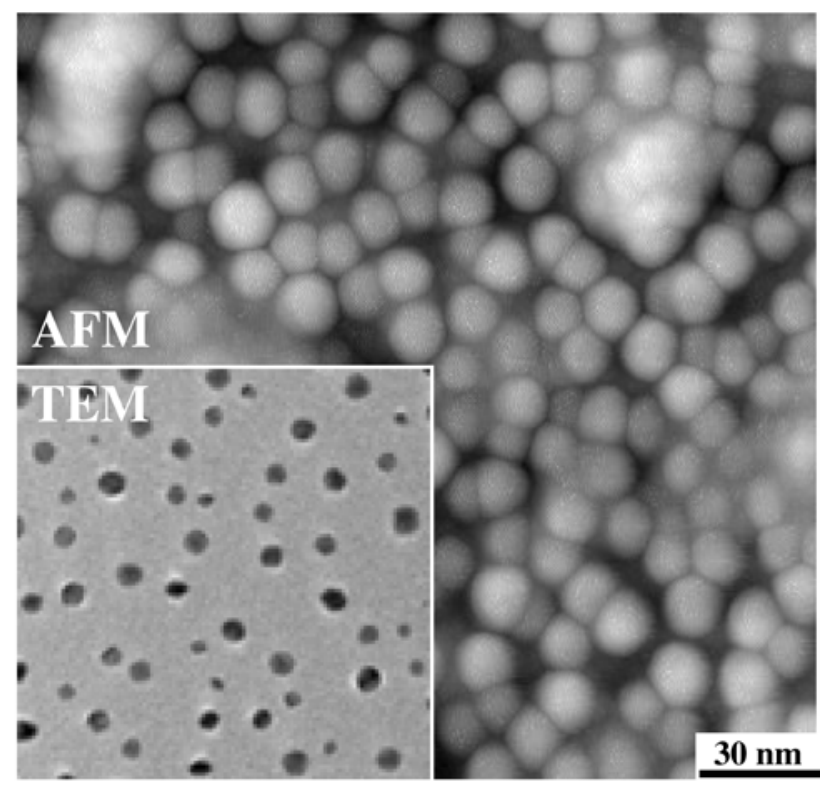

Figure 2: Topographical nc-AFM image of Pt clusters supported on an $\mathrm{Al}_{2} \mathrm{O}_{3}$ substrate, obtained with a CNT terminated probe. The inset shows a TEM image of the same surface at the same length scale. 

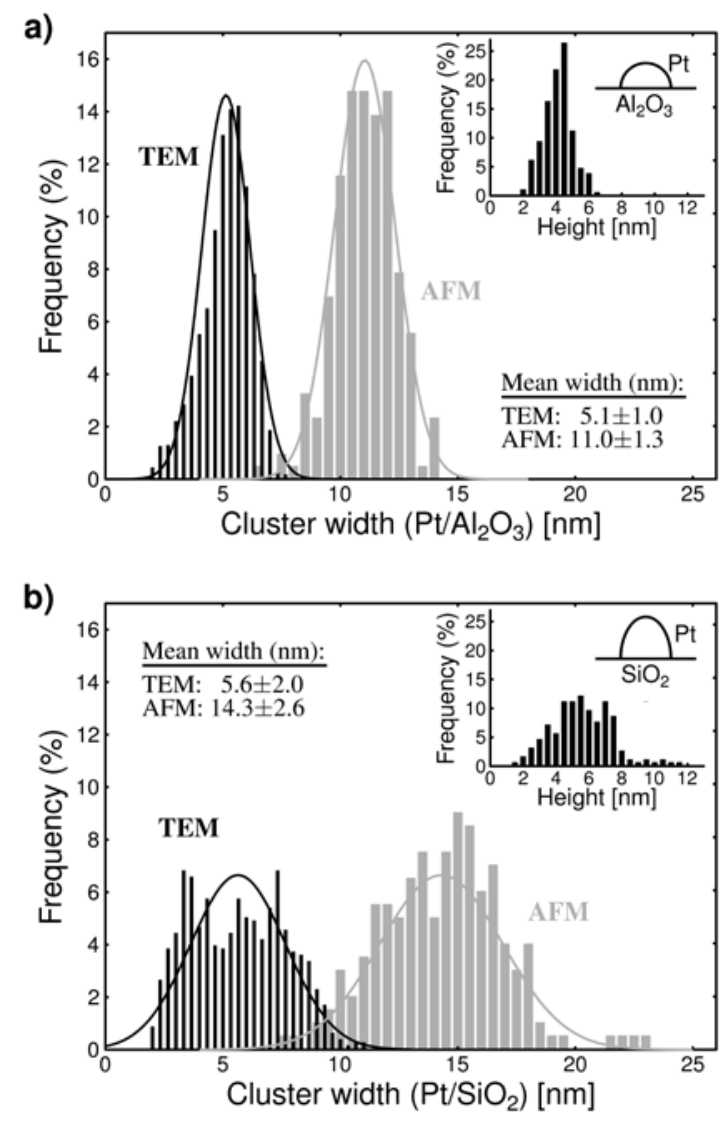

Figure 3: Width distributions for Pt nanoclusters on a) $\mathrm{Al}_{2} \mathrm{O}_{3}$ and b) $\mathrm{SiO}_{2}$, measured from both TEM (darker bars) and AFM (lighter bars) images. The solid curves represent Gaussian fits to the data, with the mean values and standard deviations indicated in each graph. The insets show the corresponding height distributions for nanoclusters from each sample, as measured with AFM, indicating that the aspect ratio between height and width is greater for nanoclusters on the $\mathrm{SiO}_{2}$ surface. 

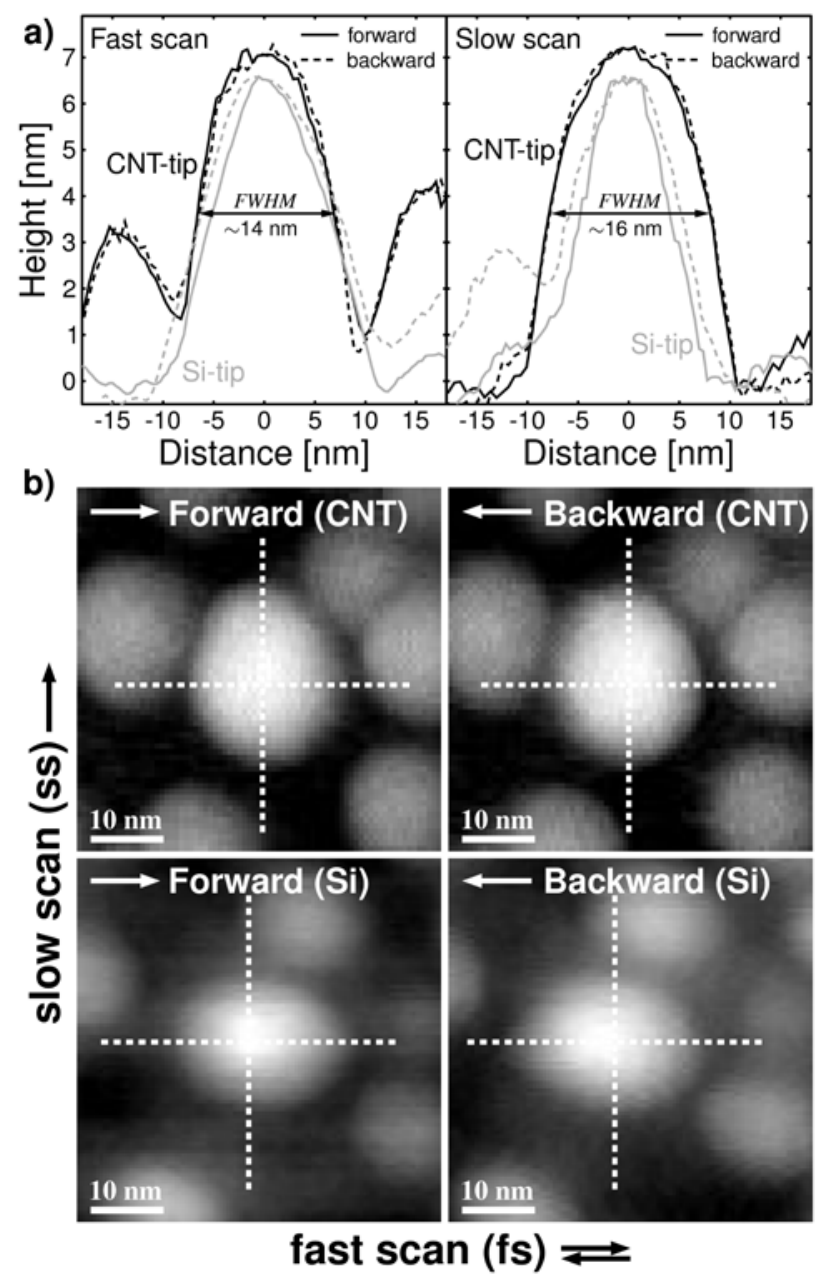

Figure 4: a) Line profiles obtained with both CNT-terminated tips and regular Si-tips, showing the profile of clusters with equal heights in both the fast scan and slow scan directions. b) nc-AFM topography images from both forward and backward scans over the same clusters. From both the line scans and the images it can be seen that tip broadening is more severe for imaging with CNT-terminated tips. Contrary to imaging with Si-tips, where tip broadening often is more severe in the fast scan direction due to limitations in the speed with which the feedback loop of the scan can react to height changes, CNT-terminated tips exhibit larger widths in the slow scan direction. 

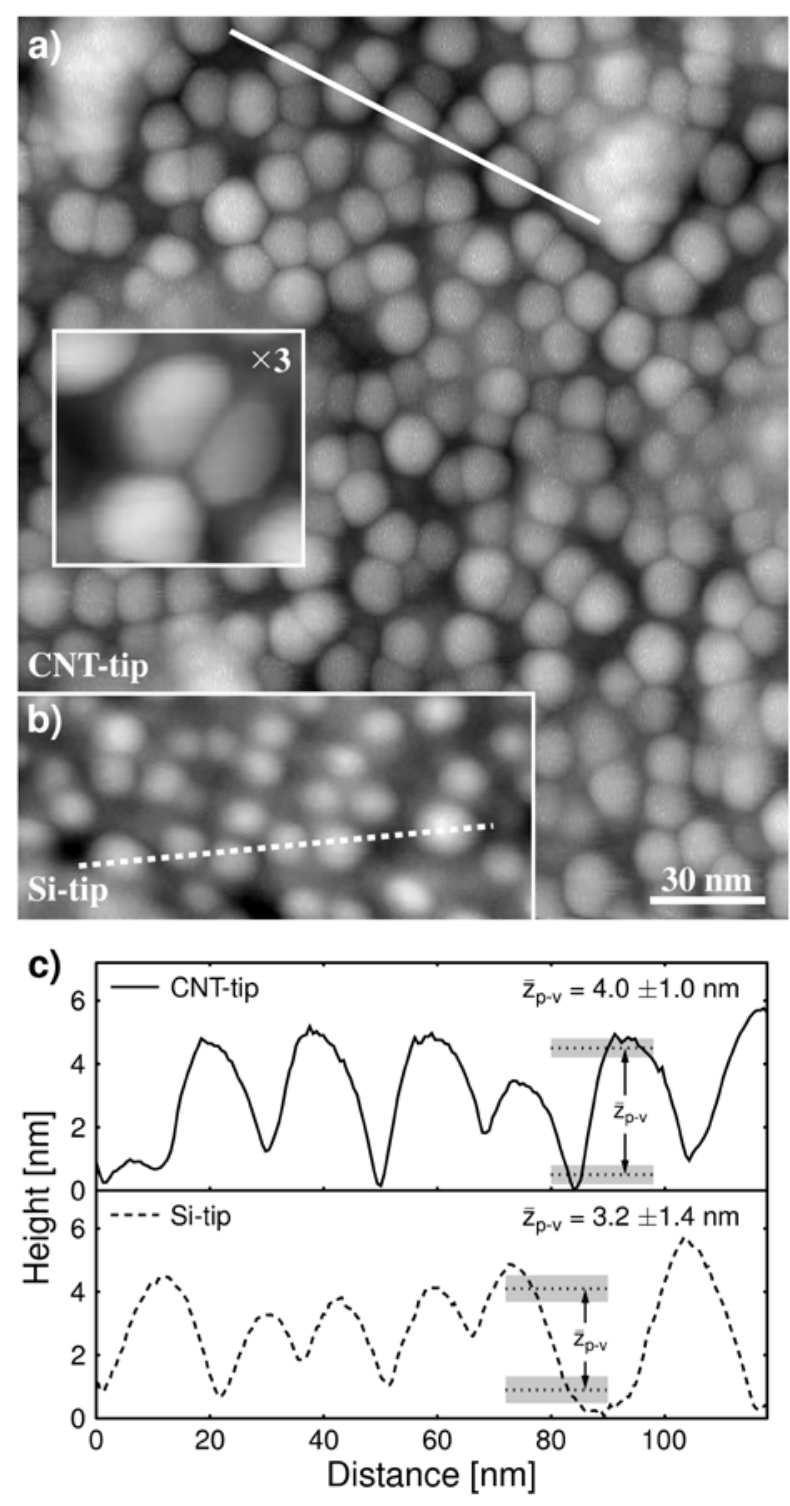

Figure 5: a) A topographical image of Pt clusters supported on an $\mathrm{Al}_{2} \mathrm{O}_{3}$ substrate obtained by nc-AFM with a CNT terminated probe. The smaller image b) shows a part of the same sample imaged with a regular Si probe. Height profiles from the lines marked in both the large image (solid line) and the small image (dashed line) are shown in c), where the advantage of CNTterminated tips is seen as peak-to-valley (p-v) distances are significantly smaller for the regular Si tip. The average peak-to-valley distance measured with each tip type is given by $z_{p-v}$, with the error indicated by the thickness of the gray lines in the graphs. The smaller inset in a) shows a magnified (x3) part of the image where the shapes of three clusters close to each other have been distorted due to a balancing of the CNTs interactions in the region between the clusters. 

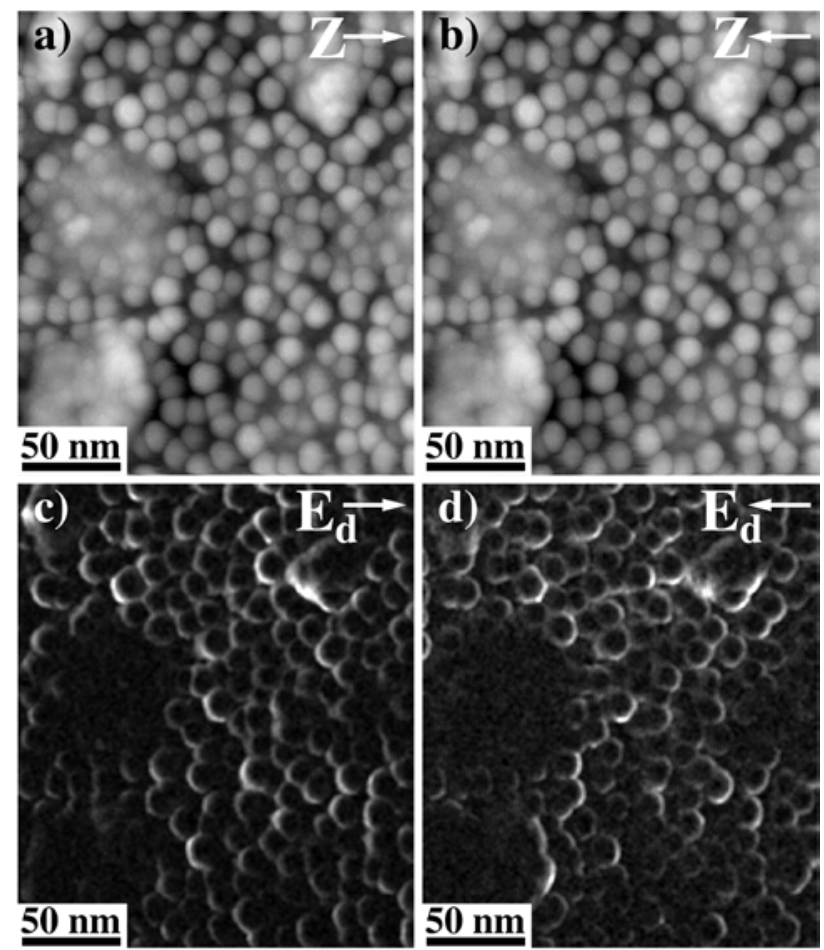

Figure 6: Topography images acquired in the a) forward and b) backward scan directions, and their corresponding dissipation signals in c) and d), respectively. A strong dissipation signal, uniformly occurring around the perimeter of each cluster and approximately independent on the scanning direction, suggests that there is an inelastic interaction which depends strongly on the properties of the CNT and the clusters and not necessarily on the scanning parameters. 


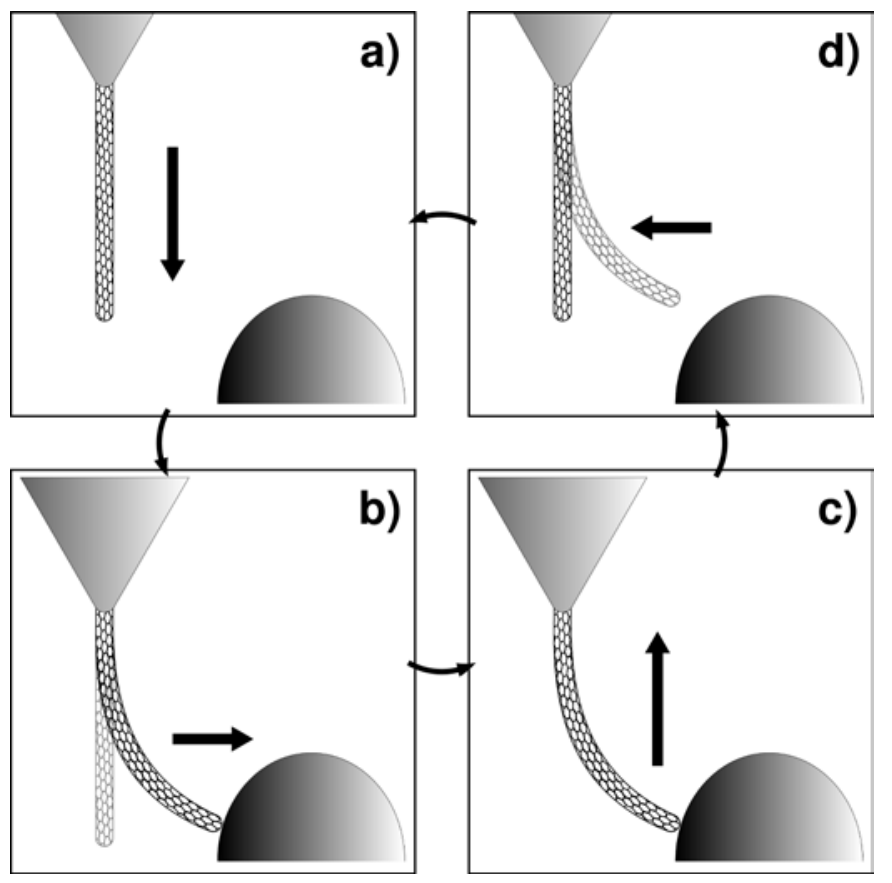

Figure 7: The motion of a CNT at the end of an SPM tip during one oscillation cycle in nc-AFM. a) The CNT is lowered toward the surface at a point close to a supported cluster. b) Attractive vdW forces bend the tube toward the cluster. If it is close enough, contact will be made between the tube and the cluster. In c) the upward motion of the tip will cause the apex of the CNT to be dragged across the surface of the cluster, therein dissipating energy from the oscillation. Finally, when the tip has been retracted far enough from the surface, in d), the CNT will bend back to its original position. The thick black arrows in each part of the figure highlight the motion of the CNT apex during the oscillation cycle. 

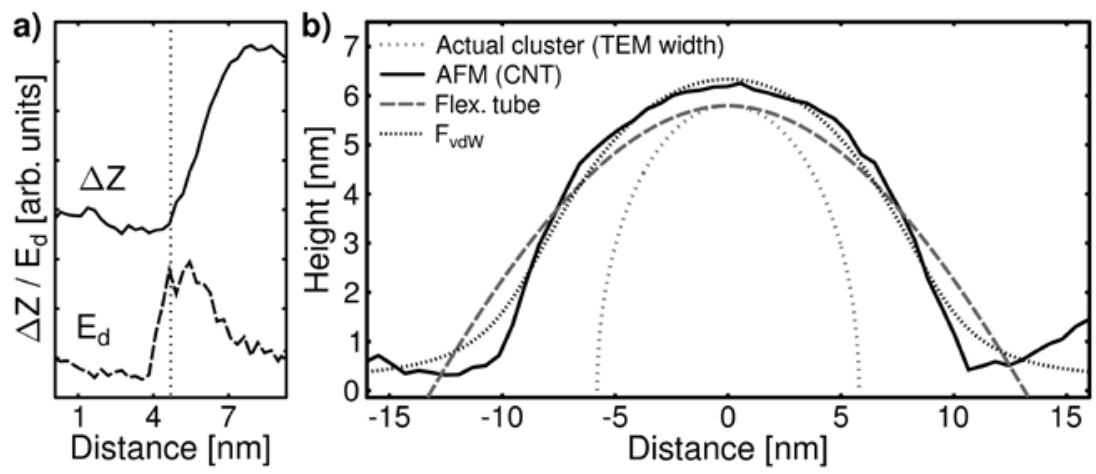

Figure 8: a) Topography ( $\Delta \mathrm{Z}$, solid line) and dissipation ( $\mathrm{E}_{\mathrm{d}}$, dashed line) signals across the same nanocluster, from nc-AFM measurements using a CNT-terminated tip. The dotted line shows how the edge of the topography signal aligns with the maximum of the dissipation signal. In b) two simple models are compared to the topography signal (solid line) across a cluster, measured with nc-AFM using a CNT-tip - a geometrical model (dashed line), where the tube is considered flexible and bending is done uniformly over its entire length, and a model that takes into account van der Waals forces ( $\mathrm{F}_{\mathrm{vdw}}$, closely dotted line). Each model is based on a cluster width calibrated by TEM measurements (sparsely dotted line) for a cluster with the same height as the experimentally measured one. The experimental topography signal is depicted here with a separation of about $0.5 \mathrm{~nm}$ from the substrate surface level and the top of the nanocluster, a distance which was typical for the tip-surface separation during the actual measurements, giving a very good fit to the curve calculated from van der Waals forces. The regions in which the force curve falls under the geometrical limit for contact between the tip and the cluster correspond very well with positions around the cluster that exhibit high measured dissipation signals. 